Pacific

Journal of

Mathematics

\title{
RESTRICTED SUCCESSIVE MINIMA
}

Martin Henk AND CARsten Thiel 


\title{
RESTRICTED SUCCESSIVE MINIMA
}

\author{
Martin Henk And CARsten ThiEL
}

\begin{abstract}
We give bounds on the successive minima of an $o$-symmetric convex body under the restriction that the lattice points realizing the successive minima are not contained in a collection of forbidden sublattices. Our investigations extend former results to forbidden full-dimensional lattices, to all successive minima and complement former results in the lower-dimensional case.
\end{abstract}

\section{Introduction}

Let $\mathscr{K}_{o}^{n}$ be the set of all $o$-symmetric convex bodies in $\mathbb{R}^{n}$ with nonempty interior, i.e., $K \in \mathcal{K}_{o}^{n}$ is an $n$-dimensional compact convex set satisfying $K=-K$. The volume, i.e., the $n$-dimensional Lebesgue measure, of a subset $X \subset \mathbb{R}^{n}$ is denoted by vol $X$. By a lattice $\Lambda \subset \mathbb{R}^{n}$ we understand a free $\mathbb{Z}$-module of rank $\operatorname{rg} \Lambda \leq n$. The set of all lattices is denoted by $\mathscr{L}^{n}$, and det $\Lambda$ denotes the determinant of $\Lambda \in \mathscr{L}^{n}$, that is the $(\operatorname{rg} \Lambda)$-dimensional volume of a fundamental cell of $\Lambda$.

For $K \in \mathscr{K}_{o}^{n}$ and $\Lambda \in \mathscr{L}^{n}$, Minkowski introduced the $i$-th successive minimum $\lambda_{i}(K, \Lambda), 1 \leq i \leq \operatorname{rg} \Lambda$, as the smallest positive number $\lambda$ such that $\lambda K$ contains at least $i$ linearly independent lattice points of $\Lambda$, i.e.,

$$
\lambda_{i}(K, \Lambda)=\min \left\{\lambda \in \mathbb{R}_{\geq 0}: \operatorname{dim}(\lambda K \cap \Lambda) \geq i\right\}, \quad 1 \leq i \leq \operatorname{rg} \Lambda .
$$

Minkowski's first fundamental theorem (see, e.g., [Gruber 2007, Sections 22-23]) on successive minima establishes an upper bound on the first successive minimum in terms of the volume of a convex body. More precisely, for $K \in \mathscr{K}_{o}^{n}$ and $\Lambda \in \mathscr{L}^{n}$ with $\operatorname{rg} \Lambda=r$, it may be formulated as

$$
\lambda_{1}(K, \Lambda)^{r} \operatorname{vol}_{r}(K \cap \operatorname{lin} \Lambda) \leq 2^{r} \operatorname{det} \Lambda,
$$

where $\operatorname{vol}_{r}(\cdot)$ denotes the $r$-dimensional volume, here with respect to the subspace $\operatorname{lin} \Lambda$, the linear hull of $\Lambda$. In the case $r=n$ we just write $\operatorname{vol}(\cdot)$. One of the many successful applications of this inequality is related to "Siegel's lemma", a catch-all term for results bounding the norm of a nontrivial lattice point lying in a linear subspace given as $\operatorname{ker} A$ where $A \in \mathbb{Z}^{m \times n}$ is an integral matrix of rank $m$. For instance, with respect to the maximum norm $|\cdot|_{\infty}$, it was shown in [Bombieri

MSC2010: primary 11H06; secondary 52C07.

Keywords: successive minima, lattice, convex body, short lattice vectors, forbidden sublattices. 
and Vaaler 1983] (see also [Ball and Pajor 1990]) that there exists a $z \in \operatorname{ker} A \backslash\{0\}$ such that

$$
|z|_{\infty} \leq \sqrt{\operatorname{det}\left(A A^{\top}\right)^{\frac{1}{n-m}}} .
$$

In fact, this follows by (1-1), where $K=[-1,1]^{n}$ is the cube of edge length 2 , $\Lambda=\operatorname{ker} A \cap \mathbb{Z}^{n}$ is an $(n-m)$-dimensional lattice of determinant at most $\sqrt{\operatorname{det}\left(A A^{\top}\right)}$, and Vaaler's result [1979] on the minimal volume of a slice of a cube, which here gives $\operatorname{vol}_{n-m}\left([-1,1]^{n} \cap \operatorname{lin} \Lambda\right) \geq 2^{n-m}$. For generalizations of Siegel's lemma to number fields we refer to [Bombieri and Vaaler 1983; Fukshansky 2006a; 2006b; Gaudron 2009; Gaudron and Rémond 2012a; Vaaler 2003].

Motivated by questions in Diophantine approximation, Fukshansky [2006a] studied an inverse problem to that addressed in Siegel's lemma, namely to bound the norm of lattice points which are not contained in the union of proper sublattices. To describe his result we need a bit more notation.

For a collection of sublattices $\Lambda_{i} \subset \Lambda, 1 \leq i \leq s$, with $\bigcup_{i=1}^{s} \Lambda_{i} \neq \Lambda$ we call

$$
\lambda_{i}\left(K, \Lambda \backslash \bigcup_{i=1}^{s} \Lambda_{i}\right)=\min \left\{\lambda \in \mathbb{R}_{\geq 0}: \operatorname{dim}\left(\lambda K \cap \Lambda \backslash \bigcup_{i=1}^{s} \Lambda_{i}\right) \geq i\right\}, \quad 1 \leq i \leq \operatorname{rg} \Lambda,
$$

the $i$-th restricted successive minimum of $K$ with respect to $\Lambda \backslash \bigcup_{i=1}^{s} \Lambda_{i}$. Observe that by the compactness of $K$ and the discreteness of $\Lambda \backslash \bigcup_{i=1}^{s} \Lambda_{i}$ these minima are well-defined. Furthermore, they behave nicely with respect to dilations, as for $\mu>0$ we have

$$
\lambda_{i}\left(\mu K, \Lambda \backslash \bigcup_{i=1}^{s} \Lambda_{i}\right)=\lambda_{i}\left(K, \frac{1}{\mu}\left(\Lambda \backslash \bigcup_{i=1}^{s} \Lambda_{i}\right)\right)=\frac{1}{\mu} \lambda_{i}\left(K, \Lambda \backslash \bigcup_{i=1}^{s} \Lambda_{i}\right) .
$$

Moreover, for a lattice $\Lambda \in \mathscr{L}^{n}, r=\operatorname{rg} \Lambda$, and a basis $\left(b_{1}, \ldots, b_{r}\right), b_{j} \in \mathbb{R}^{n}$, of $\Lambda$, let $v(\Lambda) \in \mathbb{R}^{\left(\begin{array}{c}n \\ r\end{array}\right)}$ be the vector with entries $\operatorname{det} B_{j}$, where $B_{j}$ is an $r \times r$ submatrix of $\left(b_{1}, \ldots, b_{r}\right)$. Observe that up to the order of the coordinates the vector is independent of the given basis, and on account of the Cauchy-Binet formula the Euclidean norm of $v(\Lambda)$ is the determinant of the lattice. With this notation, Fukshansky [2006a, Theorem 1.1] proved

$$
\lambda_{1}\left([-1,1]^{n}, \Lambda \backslash \bigcup_{i=1}^{s} \Lambda_{i}\right) \leq\left(\frac{3}{2}\right)^{r-1} r^{r}\left(\sum_{i=1}^{s} \frac{1}{\left|v\left(\Lambda_{i}\right)\right|_{\infty}}+\sqrt{s}\right)|v(\Lambda)|_{\infty}+1,
$$

for proper sublattices $\Lambda_{i}, 1 \leq i \leq s$, where proper means $\operatorname{rg} \Lambda_{i}<\operatorname{rg} \Lambda=r$. This result was generalized and improved in various ways in [Gaudron 2009] and [Gaudron and Rémond 2012a]. In particular, (1-3) has been extended to all $o$-symmetric bodies as well as to the adelic setting (see also [Gaudron and Rémond 2012b, Lemma 3.2] for an application). For instance, the following is a simplified version of [Gaudron 2009, 
Theorem 6.1] when we assume that $\operatorname{rg} \Lambda_{i}=\operatorname{rg} \Lambda-1=r-1$ (see also [Gaudron and Rémond 2012a, Theorem 2.2, Corollary 3.3]):

$$
\begin{aligned}
\lambda_{1}(K, \Lambda & \left.\backslash \bigcup_{i=1}^{s} \Lambda_{i}\right) \\
& \leq v \max _{1 \leq i \leq s}\left\{1, \frac{v^{r-1} \operatorname{vol}\left(K \cap \operatorname{lin} \Lambda_{i}\right)}{\omega_{r} \operatorname{det} \Lambda_{i}},\left(\frac{v}{\lambda_{1}\left(K, \Lambda \cap \operatorname{lin} \Lambda_{i}\right)}\right)^{\frac{r-2}{2}}\right\},
\end{aligned}
$$

where $v=7 r\left(s \omega_{r} \operatorname{det} \Lambda / \operatorname{vol} K\right)^{1 / r}$ and $\omega_{r}$ is the volume of the $r$-dimensional unit ball.

In our first theorem we want to complement these results on forbidden lowerdimensional lattices by a bound which takes care of the size or the structure of the individual forbidden sublattices such that the bound becomes essentially (1-1) if $\lambda_{1}\left(K, \Lambda_{i}\right) \rightarrow \infty$ for $1 \leq i \leq s$. In this case the bounds in (1-3) and (1-4) still have a dependency on $s$ of order $\sqrt{s}$ and $s^{1 / r}$, respectively. Here we have the following result.

Theorem 1.1. Let $K \in \mathscr{K}_{o}^{n}, \Lambda \in \mathscr{L}^{n}, \operatorname{rg} \Lambda=n \geq 2$, and let $\Lambda_{i} \subset \Lambda, 1 \leq i \leq s$, $\operatorname{rg} \Lambda_{i} \leq n-1$, be sublattices. Then

$$
\lambda_{1}\left(K, \Lambda \backslash \bigcup_{i=1}^{s} \Lambda_{i}\right)<6^{n-1} \frac{\operatorname{det} \Lambda}{\lambda_{1}(K, \Lambda)^{n-2} \operatorname{vol} K}\left(\sum_{i=1}^{s} \frac{1}{\lambda_{1}\left(K, \Lambda_{i}\right)}\right)+\sqrt[n]{2^{n} \frac{\operatorname{det} \Lambda}{\operatorname{vol} K}} .
$$

Note that, if $s=0$ or all the $\lambda_{1}\left(K, \Lambda_{i}\right)$ are very large, we get essentially (1-1).

Our second main theorem deals with forbidden full-dimensional sublattices those for which $\operatorname{rg} \Lambda_{i}=\operatorname{rg} \Lambda, 1 \leq i \leq s$.

Theorem 1.2. Let $K \in \mathscr{T}_{o}^{n}, \Lambda \in \mathscr{L}^{n}, \operatorname{rg} \Lambda=n \geq 2$, and let $\Lambda_{i} \subset \Lambda, 1 \leq i \leq s$, $\operatorname{rg} \Lambda_{i}=n$, be sublattices such that $\bigcup_{i=1}^{s} \Lambda_{i} \neq \Lambda$. Then

$$
\lambda_{1}\left(K, \Lambda \backslash \bigcup_{i=1}^{s} \Lambda_{i}\right)<\frac{2^{n} \operatorname{det} \Lambda}{\lambda_{1}(K, \bar{\Lambda})^{n-1} \operatorname{vol} K}\left(\sum_{i=1}^{s} \frac{\operatorname{det} \bar{\Lambda}}{\operatorname{det} \Lambda_{i}}-s+1\right)+\lambda_{1}(K, \bar{\Lambda}),
$$

where $\bar{\Lambda}=\bigcap_{i=1}^{s} \Lambda_{i}$.

In the special case $s=1$, since we may the assume $\lambda_{1}\left(K, \Lambda_{1}\right)=\lambda_{1}(K, \Lambda)$, we get the following immediate consequence:

Corollary 1.3. Let $K \in \mathscr{K}_{o}^{n}, \Lambda \in \mathscr{L}^{n}, \operatorname{rg} \Lambda=n \geq 2$, and let $\Lambda_{1} \subsetneq \Lambda, \operatorname{rg} \Lambda_{1}=n$, be a sublattice. Then

$$
\lambda_{1}\left(K, \Lambda \backslash \Lambda_{1}\right) \leq \frac{2^{n} \operatorname{det} \Lambda}{\lambda_{1}\left(K, \Lambda_{1}\right)^{n-1} \operatorname{vol} K}+\lambda_{1}(K, \Lambda) .
$$

The following example shows that the bound in Theorem 1.2 as well as the one of the corollary above cannot be improved in general by a multiplicative factor. 
Example 1.4. Let $K \in \mathscr{K} \mathcal{K}_{o}^{2}$ be the rectangle $K=[-1,1] \times[-\alpha, \alpha]$ of edge-lengths 2 and $2 \alpha, \alpha \leq 1$, and of volume $4 \alpha$. Let $\Lambda=\mathbb{Z}^{2}$, and define the sublattices

$$
\Lambda_{1}=\left\{\left(z_{1}, z_{2}\right)^{\top} \in \mathbb{Z}^{2}: z_{2} \equiv 0 \bmod 2\right\}, \quad \Lambda_{2}=\left\{\left(z_{1}, z_{2}\right)^{\top} \in \mathbb{Z}^{2}: z_{1} \equiv 0 \bmod p\right\},
$$

where $p>2$ is a prime. Then $\operatorname{det} \Lambda=1, \operatorname{det} \Lambda_{1}=2, \operatorname{det} \Lambda_{2}=p$, and

$$
\bar{\Lambda}=\Lambda_{1} \cap \Lambda_{2}=\left\{\left(z_{1}, z_{2}\right)^{\top} \in \mathbb{Z}^{2}: z_{2} \equiv 0 \bmod 2, z_{1} \equiv 0 \bmod p\right\}
$$

with $\operatorname{det} \bar{\Lambda}=2 p$. For $\alpha \leq 2 / p$ we therefore have $\lambda_{1}(K, \bar{\Lambda})=p$. Regarding the set $\Lambda \backslash\left(\Lambda_{1} \cup \Lambda_{2}\right)$, we observe that the lattice points on the axes are forbidden, but not $(1,1)^{\top}$ and so $\lambda_{1}\left(K, \Lambda \backslash\left(\Lambda_{1} \cup \Lambda_{2}\right)\right)=1 / \alpha$. Putting everything together, the bound in Theorem 1.2 evaluates for $\alpha \leq 2 / p$ to

$$
\frac{1}{\alpha}=\lambda_{1}\left(\Lambda \backslash\left(\Lambda_{1} \cup \Lambda_{2}\right)\right)<\frac{4}{p 4 \alpha}(p+1)+p=\frac{1}{\alpha}+\frac{1}{p \alpha}+p .
$$

Hence for $\alpha=2 / p^{2}$ and $p \rightarrow \infty$ the bound cannot be improved by a multiplicative factor.

In the situation of Corollary 1.3, i.e., when we consider only the forbidden lattice $\Lambda_{1}$, the upper bound in the corollary evaluates to $1 / \alpha+1$, whereas, as before, $\lambda_{1}\left(K, \Lambda \backslash \Lambda_{1}\right)=1 / \alpha$.

Before beginning with the proofs of our results we would like to mention a closely related problem, namely to cover $K \cap \Lambda, K \in \mathscr{K}_{o}^{n}$, by a minimal number $\gamma(K)$ of lattice hyperplanes. Obviously, having a $v>0$ with $\gamma(v K) \geq s+1$ implies that

$$
\lambda_{1}\left(K, \Lambda \backslash \bigcup_{i=1}^{s} \Lambda_{i}\right) \leq v
$$

in the case of lower-dimensional sublattices $\Lambda_{i}$. For bounds on $\gamma(K)$ in terms of the successive minima and other functionals from the geometry of numbers we refer to [Bárány et al. 2001; Bezdek and Hausel 1994; Bezdek and Litvak 2009].

Finally, we remark that restricted successive minima have also been investigated from an algorithmic point of view. Blömer and Naewe [2007] studied the complexity of computing $\lambda_{1}\left(K, \Lambda \backslash \bigcup_{i=1}^{s} \Lambda_{i}\right)$ for $s=1$ and when $K$ is the unit ball of an $l_{p^{-}}$ norm. They show, among other things, that some of the well-known lattice problems, like the shortest or closest lattice vector problem, are polynomial reducible to computing/approximating $\lambda_{1}\left(K, \Lambda \backslash \Lambda_{1}\right)$. Moreover, as in the case of these lattice problems an LLL-reduced basis (see [Grötschel et al. 1993, Chapter 5]) can be used to find in polynomial time a lattice vector $b$ which approximates $\lambda_{1}\left(B_{n}, \Lambda \backslash \Lambda_{1}\right)$ up to a factor of $2^{n-1}$ [Blömer and Naewe 2007, Theorem 3.6]. Here $B_{n}$ is the unit ball of the Euclidean norm. Hence, Theorem 1.1 implies (see [Grötschel et al. 1993, Theorem 5.3.13a] for a similar result in the standard setting $s=0$ ): 
Corollary 1.5. Let $\Lambda \in \mathscr{L}^{n}, \operatorname{rg} \Lambda=n \geq 2$, and let $\Lambda_{1} \subset \Lambda, \operatorname{rg} \Lambda_{1} \leq n-1$, be a sublattice. There exists a polynomial time algorithm for computing a vector $b \in \Lambda \backslash \Lambda_{1}$ of Euclidean length

$$
\|b\|<2^{n-1}\left(6^{n-1} \frac{\operatorname{det} \Lambda}{\lambda_{1}(K, \Lambda)^{n-2} \operatorname{vol} K} \frac{1}{\lambda_{1}\left(K, \Lambda_{1}\right)}+\sqrt[n]{2^{n} \frac{\operatorname{det} \Lambda}{\operatorname{vol} K}}\right) .
$$

It seems to be a challenging problem to extend this result to more than one forbidden sublattice as well as to full-dimensional forbidden lattices.

The paper is organized as follows. The proof of Theorem 1.1 will be given in the next section and full-dimensional forbidden sublattices, i.e., Theorem 1.2, will be treated in Section 3. In each of the sections we also present some extensions of the results above to higher successive minima, i.e., to $\lambda_{i}\left(K, \Lambda \backslash \bigcup_{i=1}^{s} \Lambda_{i}\right), i>1$.

\section{Avoiding lower-dimensional sublattices}

In the course of the proof we have to estimate the number of lattice points in a centrally symmetric convex body, i.e., to bound $|K \cap \Lambda|$ from below and above. Assuming $K \in \mathscr{K}_{o}^{n}$ and $\operatorname{rg} \Lambda=n$, we will use as a lower bound a classical result of van der Corput (see, e.g., [Gruber and Lekkerkerker 1987, p. 51]):

$$
|K \cap \Lambda| \geq 2\left\lfloor\frac{\operatorname{vol} K}{2^{n} \operatorname{det} \Lambda}\right\rfloor+1>\frac{\operatorname{vol} K}{2^{n-1} \operatorname{det} \Lambda}-1 .
$$

As upper bound we will use a bound in terms of the first successive minima by Betke, Henk and Wills [Betke et al. 1993]:

$$
|K \cap \Lambda| \leq\left(\frac{2}{\lambda_{1}(K, \Lambda)}+1\right)^{n} .
$$

Proof of Theorem 1.1. By scaling $K$ with $\lambda_{1}(K, \Lambda)$ we may assume without loss of generality that $\lambda_{1}(K, \Lambda)=1$, i.e., $K$ contains no nontrivial lattice point in its interior (cf. (1-2)). Let $n_{i}=\operatorname{rg} \Lambda_{i}<n$. For $\gamma \geq 1$, since $\lambda_{1}\left(K, \Lambda_{i}\right) \geq \lambda_{1}(K, \Lambda)=1$ we get, from (2-2),

$$
\left|\gamma K \backslash\{0\} \cap \Lambda_{i}\right| \leq\left(\gamma \frac{2}{\lambda_{1}\left(K, \Lambda_{i}\right)}+1\right)^{n_{i}}-1<\gamma^{n-1} 3^{n-1} \frac{1}{\lambda_{1}\left(K, \Lambda_{i}\right)} .
$$

Hence, for $\gamma \geq 1$, we have

$$
\left|\gamma K \backslash\{0\} \cap\left(\bigcup_{i=1}^{s} \Lambda_{i}\right)\right|<\gamma^{n-1} 3^{n-1} \sum_{i=1}^{s} \frac{1}{\lambda_{1}\left(K, \Lambda_{i}\right)} .
$$

Combining this bound with the upper bound (2-1) leads, for $\gamma \geq 1$, to the estimate 


$$
\begin{aligned}
\left|\gamma K \backslash\{0\} \cap \Lambda \backslash \bigcup_{i=1}^{s} \Lambda_{i}\right| & >\gamma^{n} \frac{\operatorname{vol} K}{2^{n-1} \operatorname{det} \Lambda}-2-\left|\gamma K \backslash\{0\} \cap\left(\bigcup_{i=1}^{s} \Lambda_{i}\right)\right| \\
& >\gamma^{n} \frac{\operatorname{vol} K}{2^{n-1} \operatorname{det} \Lambda}-\gamma^{n-1} 3^{n-1}\left(\sum_{i=1}^{s} \frac{1}{\lambda_{1}\left(K, \Lambda_{i}\right)}\right)-2 \\
& =\frac{\operatorname{vol} K}{2^{n-1} \operatorname{det} \Lambda}\left(\gamma^{n}-\gamma^{n-1} \beta-\rho\right),
\end{aligned}
$$

where

$$
\beta=6^{n-1} \frac{\operatorname{det} \Lambda}{\operatorname{vol} K}\left(\sum_{i=1}^{s} \frac{1}{\lambda_{1}\left(K, \Lambda_{i}\right)}\right), \quad \rho=2^{n} \frac{\operatorname{det} \Lambda}{\operatorname{vol} K} .
$$

Hence, given $\beta$ and $\rho$, we have to determine a $\gamma \geq 1$ such that $\gamma^{n}-\gamma^{n-1} \beta-\rho>0$. To this end let $\bar{\gamma}=\beta+\rho^{1 / n}$. Then

$$
\begin{aligned}
\bar{\gamma}^{n}-\bar{\gamma}^{n-1} \beta & =\left(\beta+\rho^{1 / n}\right)^{n}-\left(\beta+\rho^{1 / n}\right)^{n-1} \beta \\
& =\rho^{1 / n}\left(\beta+\rho^{1 / n}\right)^{n-1}>\rho^{1 / n} \rho^{(n-1) / n}=\rho .
\end{aligned}
$$

Finally, we observe that

$$
\bar{\gamma}>\rho^{1 / n}=\left(2^{n} \frac{\operatorname{det} \Lambda}{\operatorname{vol} K}\right)^{1 / n} \geq \lambda_{1}(K, \Lambda)=1,
$$

by (1-1) and our assumption. Hence, $\bar{\gamma}>1$ and in view of (2-5) we have $\lambda_{1}\left(K, \Lambda \backslash \bigcup_{i=1}^{s} \Lambda_{i}\right)<\bar{\gamma}$, which by the definition of $\bar{\gamma}$ yields the desired bound of the theorem with respect to our normalization $\lambda_{1}(K, \Lambda)=1$.

Compared to the bounds in (1-3) and (1-4), our formula uses only the successive minima and not the determinants of the forbidden sublattices which reflect more the structure of a lattice. However, instead of (2-2) one can use a Blichfeldt-type bound, proved in [Henze 2013], for $o$-symmetric convex bodies $K$ with $\operatorname{dim}(K \cap \Lambda)=n$; namely, if $L_{n}(x)$ is the $n$-th Laguerre polynomial,

$$
|K \cap \Lambda| \leq \frac{n !}{2^{n}} \frac{\operatorname{vol} K}{\operatorname{det} \Lambda} L_{n}(-2),
$$

This leads to a bound on $\lambda_{1}\left(K, \Lambda \backslash \bigcup_{i=1}^{s} \Lambda_{i}\right)$ where the sum over $1 / \lambda_{1}\left(K, \Lambda_{i}\right)$ is replaced by a sum over ratios of the type $\operatorname{vol}_{\operatorname{dim} H}(K \cap H) / \operatorname{det}\left(\Lambda_{i} \cap H\right)$ for certain lower-dimensional planes $H \subseteq \operatorname{lin} \Lambda_{i}$. In general, however, we have no control over the dimension of these hyperplanes $H$ nor on the volume of the sections.

Theorem 1.1 can easily be extended inductively to higher restricted successive minima $\lambda_{j+1}\left(K, \Lambda \backslash \bigcup_{i=1}^{s} \Lambda_{i}\right), 1 \leq j \leq n-1$, by avoiding, in addition, a $j$-dimensional lattice containing $j$ linearly independent lattice points corresponding to the successive minima $\lambda_{i}\left(K, \Lambda \backslash \bigcup_{i=1}^{s} \Lambda_{i}\right), 1 \leq i \leq j$. 
Corollary 2.1. Under the assumptions of Theorem 1.1 we have, for $j=1, \ldots, n-1$,

$$
\begin{aligned}
\lambda_{j+1}\left(K, \Lambda \backslash \bigcup_{i=1}^{s} \Lambda_{i}\right)<6^{n-1} & \frac{\operatorname{det} \Lambda}{\lambda_{1}(K, \Lambda)^{n-2} \operatorname{vol} K}\left(\sum_{i=1}^{s} \frac{1}{\lambda_{1}\left(K, \Lambda_{i}\right)}\right) \\
& +\left(\frac{3^{j}}{\lambda_{1}(K, \Lambda)^{j}} 2^{n-1} \frac{\operatorname{det} \Lambda}{\operatorname{vol} K}+\left(2^{n} \frac{\operatorname{det} \Lambda}{\operatorname{vol} K}\right)^{\frac{n-j}{n}}\right)^{\frac{1}{n-j}} .
\end{aligned}
$$

Proof. Let $z_{i} \in \lambda_{i}\left(K, \Lambda \backslash \bigcup_{i=1}^{s} \Lambda_{i}\right) K \cap \Lambda, 1 \leq i \leq j$, be linearly independent, and let $\bar{\Lambda}=\Lambda \cap \operatorname{lin}\left\{z_{1}, \ldots, z_{j}\right\}$. Then

$$
\lambda_{j+1}\left(K, \Lambda \backslash \bigcup_{i=1}^{s} \Lambda_{i}\right)=\lambda_{1}\left(K, \Lambda \backslash\left(\bigcup_{i=1}^{s} \Lambda_{i} \cup \bar{\Lambda}\right)\right),
$$

and we now follow the proof of Theorem 1.1. In particular, we assume $\lambda_{1}(K, \Lambda)=1$. In addition to the upper bounds on $\left|\gamma K \backslash\{0\} \cap \Lambda_{i}\right|, 1 \leq i \leq s$, in (2-3), we also use for $\gamma \geq \lambda_{1}(K, \bar{\Lambda}) \geq \lambda_{1}(K, \Lambda)=1$ the bound

$$
|\gamma K \backslash\{0\} \cap \bar{\Lambda}|<\left(\frac{2 \gamma}{\lambda_{1}(K, \bar{\Lambda})}+1\right)^{j} \leq 3^{j}\left(\frac{\gamma}{\lambda_{1}(K, \bar{\Lambda})}\right)^{j} .
$$

Combining this bound with $(2-1)$ leads for $\gamma \geq \lambda_{1}(K, \bar{\Lambda})$ to

$$
\begin{aligned}
\mid \gamma K \backslash\{0\} \cap & \Lambda \backslash\left(\bigcup_{i=1}^{s} \Lambda_{i} \cup \bar{\Lambda}\right) \mid \\
& >\gamma^{n} \frac{\operatorname{vol} K}{2^{n-1} \operatorname{det} \Lambda}-2-\left|\gamma K \backslash\{0\} \cap\left(\bigcup_{i=1}^{s} \Lambda_{i}\right)\right|-|\gamma K \backslash\{0\} \cap \bar{\Lambda}| \\
& >\gamma^{n} \frac{\operatorname{vol} K}{2^{n-1} \operatorname{det} \Lambda}-2-\gamma^{n-1} 3^{n-1}\left(\sum_{i=1}^{s} \frac{1}{\lambda_{1}\left(K, \Lambda_{i}\right)}\right)-3^{j}\left(\frac{\gamma}{\lambda_{1}(K, \bar{\Lambda})}\right)^{j} \\
& =\frac{\operatorname{vol} K}{2^{n-1} \operatorname{det} \Lambda}\left(\gamma^{n}-\gamma^{n-1} \beta-\gamma^{j} \alpha-\rho\right),
\end{aligned}
$$

with

$\beta=6^{n-1} \frac{\operatorname{det} \Lambda}{\operatorname{vol} K}\left(\sum_{i=1}^{s} \frac{1}{\lambda_{1}\left(K, \Lambda_{i}\right)}\right), \quad \alpha=\frac{3^{j}}{\lambda_{1}(K, \bar{\Lambda})^{j}} 2^{n-1} \frac{\operatorname{det} \Lambda}{\operatorname{vol} K}, \quad \rho=2^{n} \frac{\operatorname{det} \Lambda}{\operatorname{vol} K}$. Now setting $\bar{\gamma}=\beta+\left(\alpha+\rho^{\frac{n-j}{n}}\right)^{\frac{1}{n-j}}$ we see as in the proof of Theorem 1.1 that

$$
\begin{aligned}
\bar{\gamma}^{n}-\bar{\gamma}^{n-1} \beta-\bar{\gamma}^{j} \alpha-\rho & =\bar{\gamma}^{j}\left(\bar{\gamma}^{n-j}-\beta \bar{\gamma}^{n-j-1}-\alpha\right)-\rho \\
& >\bar{\gamma}^{j} \rho^{(n-j) / n}-\rho>0 .
\end{aligned}
$$

Since $\bar{\gamma}>\beta+\rho^{1 / n}$, which is, by the proof of Theorem 1.1, an upper bound on $\lambda_{1}(K, \bar{\Lambda})$, we also have $\bar{\gamma}>\lambda_{1}(K, \bar{\Lambda})$ and so we know $\lambda_{j+1}\left(K, \Lambda \backslash \bigcup_{i=1}^{s} \Lambda_{i}\right)<\bar{\gamma}$, 
by (2-8). By the definition of $\bar{\gamma}$ we get the required upper bound with respect to the normalization $\lambda_{1}(K, \Lambda)=1$.

An upper bound on $\lambda_{j}\left(K, \Lambda \backslash \bigcup_{i=1}^{s} \Lambda_{i}\right)$ of a different kind involves the so-called covering radius $\mu(K, \Lambda)$ of a convex body $K \in \mathscr{Y}_{o}^{n}$ and a lattice $\Lambda \in \mathscr{L}^{n}, \operatorname{rg} \Lambda=n$. This is the smallest positive number $\mu$ such that any translate of $\mu K$ contains a lattice point:

$$
\mu(K, \Lambda)=\min \left\{\mu>0:(t+\mu K) \cap \Lambda \neq \varnothing \text { for all } t \in \mathbb{R}^{n}\right\} .
$$

(see [Gruber and Lekkerkerker 1987, Chapter 2, Section 13]).

Proposition 2.2. Under the assumptions of Theorem 1.1 we have

$$
\lambda_{1}\left(K, \Lambda \backslash \bigcup_{i=1}^{s} \Lambda_{i}\right) \leq(s+1) \mu(K, \Lambda),
$$

and hence $\lambda_{j}\left(K, \Lambda \backslash \bigcup_{i=1}^{s} \Lambda_{i}\right) \leq(s+2) \mu(K, \Lambda)$ for $2 \leq j \leq n$.

Proof. Observe that on account of (2-6) the bound for $j \geq 2$ follows from the one for $\lambda_{1}\left(K, \Lambda \backslash \bigcup_{i=1}^{s} \Lambda_{i}\right)$. For the proof in the case $j=1$ let $H_{i}=\operatorname{lin} \Lambda_{i}, 1 \leq i \leq s$, and for brevity we write $\bar{\mu}$ instead of $\mu(K, \Lambda)$. By Ball's solution [1991] of the affine plank problem for $o$-symmetric convex bodies, applied to $\bar{\mu} K$, we know that there exists a $t \in \mathbb{R}^{n}$ such that

$$
\left(t+\frac{1}{s+1} \bar{\mu} K\right) \subset \bar{\mu} K \quad \text { and } \quad \operatorname{int}\left(t+\frac{1}{s+1} \bar{\mu} K\right) \cap H_{i}=\varnothing, 1 \leq i \leq s,
$$

where $\operatorname{int}(\cdot)$ denotes the interior. Thus, for any $\epsilon>0$ the body $(s+1+\epsilon) \bar{\mu} K$ contains a translate $t_{\epsilon}+\bar{\mu} K$ having no points in common with $H_{i}, 1 \leq i \leq s$. Hence, together with the definition of the covering radius, we have $\left(t_{\epsilon}+\bar{\mu} K\right) \cap \Lambda \backslash \bigcup_{i=1}^{s} \Lambda_{i} \neq \varnothing$ and so $\lambda_{1}\left(K, \Lambda \backslash \bigcup_{i=1}^{s} \Lambda_{i}\right) \leq(s+1+\epsilon) \bar{\mu}$. By the arbitrariness of $\epsilon$ and the compactness of $K$ the assertion follows.

For a comparable uniform bound in the much more general adelic setting and, of course, with a completely different method see [Gaudron and Rémond 2012a, Proposition 3.2].

\section{Avoiding full-dimensional sublattices}

If the forbidden sublattices are full-dimensional we cannot argue as in the lowerdimensional case, since now the number of forbidden lattice points in $\lambda K \cap \bigcup_{i=1}^{s} \Lambda_{i}$ grows with the same order of magnitude with respect to $\lambda$ as the number of points in $\lambda K \cap \Lambda$.

The tool we use in this full-dimensional case is the torus group $\mathbb{R}^{n} / \bar{\Lambda}$ for a certain lattice $\bar{\Lambda}$. For a more detailed discussion we refer to [Gruber 2007, Section 26]. 
We recall that this quotient of abelian groups is a compact topological group and we may identify $\mathbb{R}^{n} / \bar{\Lambda}$ with a fundamental parallelepiped $P$ of $\bar{\Lambda}$ :

$$
\mathbb{R}^{n} / \bar{\Lambda} \sim P=\left\{\rho_{1} b_{1}+\cdots+\rho_{n} b_{n}: 0 \leq \rho_{i}<1\right\},
$$

where $b_{1}, \ldots, b_{n}$ form a basis of $\bar{\Lambda}$. Hence for $X \subset \mathbb{R}^{n}$, the set $X$ modulo $\bar{\Lambda}, X / \bar{\Lambda}$, can be described as

$$
X / \bar{\Lambda}=\{y \in P: \exists b \in \bar{\Lambda} \text { such that } y+b \in X\}=(\bar{\Lambda}+X) \cap P,
$$

and we can think of $\bar{X} \subseteq \mathbb{R}^{n} / \bar{\Lambda}$ as its image under inclusion into $\mathbb{R}^{n}$. In the same spirit we may identify addition $\oplus$ in $\mathbb{R}^{n} / \bar{\Lambda}$ with the corresponding operation in $\mathbb{R}^{n}$, i.e., for $\bar{X}_{1}, \bar{X}_{2} \subset \mathbb{R}^{n} / \bar{\Lambda}$ we have

$$
\bar{X}_{1} \oplus \bar{X}_{2}=\left(\left(\bar{X}_{1}+\bar{X}_{2}\right)+\bar{\Lambda}\right) \cap P .
$$

As $\mathbb{R}^{n} / \bar{\Lambda}$ is a compact abelian group, there is a unique Haar measure $\operatorname{vol}_{T}(\cdot)$ on it normalized to $\operatorname{vol}_{T}\left(\mathbb{R}^{n} / \bar{\Lambda}\right)=\operatorname{det} \bar{\Lambda}$, and for a "nice" measurable set $X \subset \mathbb{R}^{n}$ or $\bar{X} \subset \mathbb{R}^{n} / \bar{\Lambda}$ we have

$$
\operatorname{vol}_{T}(X / \bar{\Lambda})=\operatorname{vol}((\bar{\Lambda}+X) \cap P) \quad \text { and } \quad \operatorname{vol}_{T}(\bar{X})=\operatorname{vol}((\bar{\Lambda}+\bar{X}) \cap P) .
$$

Regarding the volume of the sum of two sets $\bar{X}_{1}, \bar{X}_{2} \subset \mathbb{R}^{n} / \bar{\Lambda}$ we have the following classical sum theorem of Kneser and Macbeath [Gruber 2007, Theorem 26.1]:

$$
\operatorname{vol}_{T}\left(\bar{X}_{1} \oplus \bar{X}_{2}\right) \geq \min \left\{\operatorname{vol}_{T}\left(\bar{X}_{1}\right)+\operatorname{vol}_{T}\left(\bar{X}_{2}\right), \operatorname{det} \bar{\Lambda}\right\} .
$$

We also note that for an $o$-symmetric convex body $K \in \mathscr{Y}_{o}^{n}$ and $\lambda \geq 0$ the set $\bar{\Lambda}+\lambda K$ forms a lattice packing, i.e., for any two different lattice points $\bar{a}, \bar{b} \in \bar{\Lambda}$ the translates $\bar{a}+\lambda K$ and $\bar{b}+\lambda K$ do not overlap if and only if $\lambda \leq \lambda_{1}(K, \bar{\Lambda}) / 2$. Hence we know that, for $0 \leq \lambda \leq \lambda_{1}(K, \bar{\Lambda}) / 2$,

$$
\operatorname{vol}_{T}(\lambda K / \bar{\Lambda})=\operatorname{vol}((\lambda K+\bar{\Lambda}) \cap P)=\lambda^{n} \operatorname{vol} K .
$$

Furthermore, we also need a "torus version" of van der Corput's result (2-1):

Lemma 3.1. Let $K \in \mathscr{K}_{o}^{n}, \Lambda \in \mathscr{L}^{n}, \operatorname{rg} \Lambda=n$ and let $\bar{\Lambda} \subsetneq \Lambda$ be a sublattice with $\operatorname{rg} \bar{\Lambda}=n$, and let $m \in \mathbb{N}$ with $m \operatorname{det} \Lambda<\operatorname{det} \bar{\Lambda}$. If $\operatorname{vol}_{T}\left(\frac{1}{2} K / \bar{\Lambda}\right) \geq m \operatorname{det} \Lambda$ then

$$
\#(K / \bar{\Lambda} \cap \Lambda) \geq m+1 ;
$$

i.e., $K$ contains at least $m+1$ lattice points of $\Lambda$ belonging to different cosets modulo $\bar{\Lambda}$.

Proof. By the compactness of $K$ and the discreteness of lattices we may assume $\operatorname{vol}_{T}\left(\frac{1}{2} K / \bar{\Lambda}\right)>m \operatorname{det} \Lambda$. Let $P$ be a fundamental parallelepiped of the lattice $\bar{\Lambda}$. Then by assumption we have for the measurable set $X=\left(\frac{1}{2} K+\bar{\Lambda}\right) \cap P$ that vol $X>m$ det $\Lambda$. According to a result of van der Corput [Gruber and Lekkerkerker 
1987, Section 6.1, Theorem 1] we know that there exists pairwise different $x_{i} \in X$, $1 \leq i \leq m+1$, such that $x_{i}-x_{j} \in \Lambda$. By the $o$-symmetry and convexity of $K$ we have $(X-X)=(K+\bar{\Lambda}) \cap(P-P)$, and since $(P-P) \cap \bar{\Lambda}=\{0\}$ we conclude that

$$
x_{i}-x_{j} \in(K+\bar{\Lambda}) \cap \Lambda \backslash \bar{\Lambda}, \quad i \neq j .
$$

Hence the $m$ points $x_{i}-x_{1} \in K+\bar{\Lambda}, i=2, \ldots, m+1$, belong to different nontrivial cosets of $\Lambda$ modulo $\bar{\Lambda}$ and thus $\#(K / \bar{\Lambda} \cap \Lambda) \geq m+1$, where the additional 1 counts the origin.

We now state some simple facts on the intersection of full-dimensional sublattices.

Lemma 3.2. Let $\Lambda \in \mathscr{L}^{n}, \Lambda_{i} \subseteq \Lambda, 1 \leq i \leq s, \operatorname{rg} \Lambda_{i}=\operatorname{rg} \Lambda=n$, and let $\bar{\Lambda}=\bigcap_{i=1}^{s} \Lambda_{i}$. Then $\bar{\Lambda} \in \mathscr{L}^{n}$ with $\operatorname{rg} \bar{\Lambda}=n$, and

$$
\max _{1 \leq i \leq s} \operatorname{det} \Lambda_{i} \leq \operatorname{det} \bar{\Lambda} \leq(\operatorname{det} \Lambda)^{1-s}\left(\operatorname{det} \Lambda_{1}\right) \cdots\left(\operatorname{det} \Lambda_{s}\right) .
$$

Moreover, with $m=\sum_{i=1}^{s} \operatorname{det} \bar{\Lambda} / \operatorname{det} \Lambda_{i}-s+1$ we have:

(i) The union $\bigcup_{i=1}^{s} \Lambda_{i}$ is covered by at most $m$ cosets of $\Lambda$ modulo $\bar{\Lambda}$.

(ii) If $\operatorname{det} \bar{\Lambda} / \operatorname{det} \Lambda \geq m+1$ then $\Lambda \neq \bigcup_{i=1}^{s} \Lambda_{i}$.

Proof. In order to show that $\bar{\Lambda}$ is a full-dimensional lattice it suffices to consider $s=2$. Obviously, $\Lambda_{1} \cap \Lambda_{2}$ is a discrete subgroup of $\Lambda$ and it also contains $n$ linearly independent points, e.g., $\left(\operatorname{det} \Lambda_{2}\right) a_{1}, \ldots,\left(\operatorname{det} \Lambda_{2}\right) a_{n}$, where $a_{1}, \ldots, a_{n}$ is a basis of $\Lambda_{1}$. Hence $\bar{\Lambda}$ is a full-dimensional lattice; see [Gruber and Lekkerkerker 1987, Section 3.2, Theorem 2]. The lower bound on $\operatorname{det} \bar{\Lambda}$ is clear by the inclusion $\bar{\Lambda} \subseteq \Lambda_{i}, 1 \leq i \leq s$. For the upper bound we observe that two points $g, h \in \Lambda$ belong to different cosets modulo $\bar{\Lambda}$ if and only if $g$ and $h$ belong to different cosets of $\Lambda$ modulo at least one $\Lambda_{i}$. There are $\operatorname{det} \Lambda_{i} / \operatorname{det} \Lambda$ many cosets for each $i$ and so we get the upper bound.

For (i) we note that since $\Lambda_{i}$ is the union of $\operatorname{det} \bar{\Lambda} / \operatorname{det} \Lambda_{i}$ many cosets modulo $\bar{\Lambda}$, the union is certainly covered by $\sum_{i=1}^{s} \operatorname{det} \bar{\Lambda} / \operatorname{det} \Lambda_{i}=m+s-1$ many cosets of $\Lambda$ modulo $\bar{\Lambda}$. But here we have counted the trivial coset at least $s$ times. Part (ii) is a direct consequence of part (i).

Lemma 3.2(ii) implies, in particular, that the union of two strict sublattices can never be the whole lattice. This is no longer true for three sublattices, as we see in the next example, which also shows that Lemma 3.2(ii) is not an equivalence.

Example 3.3. Let $\Lambda=\mathbb{Z}^{2}$, and let $\Lambda_{1}, \ldots, \Lambda_{4} \subset \mathbb{Z}^{2}$ be the sublattices

$$
\begin{array}{ll}
\Lambda_{1}=\left\{\left(z_{1}, z_{2}\right)^{\top} \in \mathbb{Z}^{2}: z_{2} \equiv 0 \bmod 2\right\}, & \Lambda_{2}=\left\{\left(z_{1}, z_{2}\right)^{\top} \in \mathbb{Z}^{2}: z_{1} \equiv 0 \bmod 2\right\}, \\
\Lambda_{3}=\left\{\left(z_{1}, z_{2}\right)^{\top} \in \mathbb{Z}^{2}: z_{2} \equiv 0 \bmod 3\right\}, & \Lambda_{4}=\left\{\left(z_{1}, z_{2}\right)^{\top} \in \mathbb{Z}^{2}: z_{1} \equiv z_{2} \bmod 2\right\} .
\end{array}
$$


Then $\Lambda_{1} \cup \Lambda_{2} \cup \Lambda_{4}=\Lambda$ but $\Lambda_{1} \cup \Lambda_{2} \cup \Lambda_{3} \neq \Lambda$. Furthermore $\operatorname{det} \Lambda=1$, $\operatorname{det} \Lambda_{1}=\operatorname{det} \Lambda_{2}=\operatorname{det} \Lambda_{4}=2, \operatorname{det} \Lambda_{3}=3$ and

$$
\bar{\Lambda}=\Lambda_{1} \cap \Lambda_{2} \cap \Lambda_{3}=\left\{\left(z_{1}, z_{2}\right)^{\top} \in \mathbb{Z}^{2}: z_{1} \equiv 0 \bmod 2, z_{2} \equiv 0 \bmod 6\right\}
$$

with $\operatorname{det} \bar{\Lambda}=12$, while $\sum_{i=1}^{3} \frac{\operatorname{det} \bar{\Lambda}}{\operatorname{det} \Lambda_{i}}-1=15$.

We now come to the proof of the full-dimensional case.

Proof of Theorem 1.2. Let $\Lambda_{1}, \ldots, \Lambda_{s}$ be the full-dimensional forbidden sublattices of the given lattice $\Lambda$ and let $\bar{\Lambda}=\bigcap_{i=1}^{s} \Lambda_{i}$. Let

$$
m=\min \left\{\sum_{i=1}^{s} \frac{\operatorname{det} \bar{\Lambda}}{\operatorname{det} \Lambda_{i}}-s+1, \frac{\operatorname{det} \bar{\Lambda}}{\operatorname{det} \Lambda}\right\} .
$$

Claim 1. Let $\lambda>0$ with $\operatorname{vol}_{T}\left(\left(\lambda \frac{1}{2} K\right) / \bar{\Lambda}\right) \geq m \operatorname{det} \Lambda$. Then

$$
\lambda_{1}\left(K, \Lambda \backslash \bigcup_{i=1}^{s} \Lambda_{i}\right) \leq \lambda
$$

To verify the claim, we first assume

$$
m=\sum_{i=1}^{s} \frac{\operatorname{det} \bar{\Lambda}}{\operatorname{det} \Lambda_{i}}-s+1<\frac{\operatorname{det} \bar{\Lambda}}{\operatorname{det} \Lambda} .
$$

By Lemma 3.1, $\lambda K$ contains $m+1$ lattice points of $\Lambda$ belonging to different cosets with respect to $\bar{\Lambda}$. By Lemma 3.2 (i), $\bigcup_{i=1}^{s} \Lambda_{i}$ is covered by at most $m$ cosets of $\Lambda$ modulo $\bar{\Lambda}$, and thus $\lambda K$ contains a lattice point of $\Lambda \backslash \bigcup_{i=1}^{s} \Lambda_{i}$.

Next suppose that $m=\operatorname{det} \bar{\Lambda} / \operatorname{det} \Lambda$. Then

$$
\operatorname{vol}_{T}\left(\left(\lambda \frac{1}{2} K\right) / \bar{\Lambda}\right)=\operatorname{det} \bar{\Lambda}=\operatorname{vol}_{T}\left(\mathbb{R}^{n} / \bar{\Lambda}\right)
$$

and, in particular, $\lambda K$ contains a representative of each coset of $\Lambda$ modulo $\bar{\Lambda}$. By assumption there exists a coset containing a point $a \in \Lambda \backslash \bigcup_{i=1}^{s} \Lambda_{i}$, and hence all points of this coset, that is $a+\bar{\Lambda}$, lie in $\Lambda \backslash \bigcup_{i=1}^{s} \Lambda_{i}$.

This verifies the claim and it remains to compute a $\lambda$ with

$$
\operatorname{vol}_{T}\left(\left(\lambda \frac{1}{2} K\right) / \bar{\Lambda}\right) \geq m \operatorname{det} \Lambda \text {. }
$$

To this end we set $\lambda_{1}=\lambda_{1}(K, \bar{\Lambda})$ and we write an arbitrary $\lambda>0$ modulo $\lambda_{1}$ in the form $\lambda=\left\lfloor\lambda / \lambda_{1}\right\rfloor \lambda_{1}+\rho \lambda_{1}$, with $0 \leq \rho<1$. Hence, in view of the sum theorem of Kneser and Macbeath (3-1) and the packing property (3-2) of $\lambda_{1}$ with respect to $\frac{1}{2} K$, we may write 


$$
\begin{aligned}
& \operatorname{vol}_{T}\left(\left(\lambda \frac{1}{2} K\right) / \bar{\Lambda}\right)=\operatorname{vol}_{T}\left(\left(\left(\left\lfloor\frac{\lambda}{\lambda_{1}}\right\rfloor \frac{\lambda_{1}}{2}+\rho \frac{\lambda_{1}}{2}\right) K\right) / \bar{\Lambda}\right) \\
& =\operatorname{vol}_{T}(\underbrace{\left(\frac{\lambda_{1}}{2} K\right) / \bar{\Lambda} \oplus \cdots \oplus\left(\frac{\lambda_{1}}{2} K\right) / \bar{\Lambda}}_{\left\lfloor\lambda / \lambda_{1}\right\rfloor} \oplus\left(\frac{\rho \lambda_{1}}{2} K\right) / \bar{\Lambda}) \\
& \geq \min \left\{\left(\left\lfloor\frac{\lambda}{\lambda_{1}}\right\rfloor+\rho^{n}\right)\left(\frac{\lambda_{1}}{2}\right)^{n} \operatorname{vol} K, \operatorname{det} \bar{\Lambda}\right\} \text {. }
\end{aligned}
$$

Thus, (3-3) is certainly satisfied for a $\bar{\lambda}$ with

$$
\left(\left\lfloor\frac{\bar{\lambda}}{\lambda_{1}}\right\rfloor+\rho^{n}\right)\left(\frac{\lambda_{1}}{2}\right)^{n} \operatorname{vol} K=\left(\sum_{i=1}^{s} \frac{\operatorname{det} \bar{\Lambda}}{\operatorname{det} \Lambda_{i}}-s+1\right) \operatorname{det} \Lambda .
$$

Using that

$$
\left\lfloor\frac{\lambda}{\lambda_{1}}\right\rfloor+\rho^{n}>\frac{\lambda-\lambda_{1}}{\lambda_{1}},
$$

we find

$$
\lambda_{1}\left(K, \Lambda \backslash \bigcup_{i=1}^{s} \Lambda_{i}\right) \leq \bar{\lambda}<\frac{2^{n} \operatorname{det} \Lambda}{\lambda_{1}^{n-1} \operatorname{vol} K}\left(\sum_{i=1}^{s} \frac{\operatorname{det} \bar{\Lambda}}{\operatorname{det} \Lambda_{i}}-s+1\right)+\lambda_{1} .
$$

Remark 3.4. The bound in Theorem 1.2 can be slightly improved in lower dimensions by noticing that in (3-5) we may replace $\left(\lambda-\lambda_{1}\right) / \lambda_{1}$ by $\lambda / \lambda_{1}-\rho+\rho^{n}$. Since $\rho-\rho^{n}$ takes its maximum at $\rho=(1 / n)^{1 /(n-1)}$ we get in this way

$$
\begin{aligned}
\lambda_{1}(K, \Lambda & \left.\backslash \bigcup_{i=1}^{s} \Lambda_{i}\right) \\
& \leq \frac{2^{n} \operatorname{det} \Lambda}{\lambda_{1}(K, \bar{\Lambda})^{n-1} \operatorname{vol} K}\left(\sum_{i=1}^{s} \frac{\operatorname{det} \bar{\Lambda}}{\operatorname{det} \Lambda_{i}}-s+1\right)+n^{-1 /(n-1)} \frac{n-1}{n} \lambda_{1}(K, \bar{\Lambda}) .
\end{aligned}
$$

There is a straightforward way to extend Theorem 1.2 to higher successive minima which we will first present in the special case $s=1$.

Corollary 3.5. Under the assumptions of Corollary 1.3 we have, for $1 \leq i \leq n$,

$$
\lambda_{i}\left(K, \Lambda \backslash \Lambda_{1}\right) \leq \frac{2^{n} \operatorname{det} \Lambda}{\lambda_{1}\left(K, \Lambda_{1}\right)^{n-1} \operatorname{vol} K}+\lambda_{1}(K, \Lambda)+\lambda_{i}(K, \Lambda) .
$$

Proof. By Corollary 1.3 it suffices to show $\lambda_{i}\left(K, \Lambda \backslash \Lambda_{1}\right) \leq \lambda_{1}\left(K, \Lambda \backslash \Lambda_{1}\right)+\lambda_{i}(K, \Lambda)$ for $i=2, \ldots, n$. To this end let $a \in \lambda_{1}\left(K, \Lambda \backslash \Lambda_{1}\right) K \cap \Lambda \backslash \Lambda_{1}$ and let $b_{1}, \ldots, b_{n}$ be linearly independent with $b_{j} \in \lambda_{j}(K, \Lambda) K \cap \Lambda, j=1, \ldots, n$. Since not both $b_{j}$ and $a+b_{j}$ can belong to the forbidden sublattice $\Lambda_{1}$ we can select from each pair $b_{j}, a+b_{j}$ one contained in $\Lambda \backslash \Lambda_{1}, 1 \leq j \leq n$. Let these points be denoted by $\bar{b}_{j}, j=1, \ldots, n$. Then $a, \bar{b}_{j} \in\left(\lambda_{1}\left(K, \Lambda \backslash \Lambda_{1}\right)+\lambda_{j}(K, \Lambda)\right) K, 1 \leq j \leq n$.

Now choose $k$ such that $a \notin \operatorname{lin}\left(\left\{b_{1}, \ldots, b_{n}\right\} \backslash\left\{b_{k}\right\}\right)$. Then the lattice points $a, \bar{b}_{1}, \ldots \bar{b}_{k-1}, \bar{b}_{k+1}, \ldots, \bar{b}_{n}$ are linearly independent and we are done. 
For $s>1$ the excluded substructure $\bigcup_{i=1}^{s} \Lambda_{i}$ is, in general, not a lattice anymore and so we cannot argue as above. Therefore, in this case, we choose the vectors $b_{j}$, $1 \leq j \leq n$, from the lattice $\bar{\Lambda}=\bigcap_{i=1}^{s} \Lambda_{i}$. Then for $a \in \Lambda \backslash \bigcup_{i=1}^{s} \Lambda_{i}$ we have

$$
a, a+b_{1}, a+b_{2}, \ldots, a+b_{n} \in \Lambda \backslash \bigcup_{i=1}^{s} \Lambda_{i},
$$

and analogously to the proof of Corollary 3.5 we get:

Corollary 3.6. Under the assumptions of Theorem 1.2 we have, for $1 \leq i \leq n$,

$$
\begin{aligned}
\lambda_{i}\left(K, \Lambda \backslash \bigcup_{i=1}^{s} \Lambda_{i}\right) & \\
& \leq \frac{2^{n} \operatorname{det} \Lambda}{\lambda_{1}(K, \bar{\Lambda})^{n-1} \operatorname{vol} K}\left(\sum_{i=1}^{s} \frac{\operatorname{det} \bar{\Lambda}}{\operatorname{det} \Lambda_{i}}-s+1\right)+\lambda_{1}(K, \bar{\Lambda})+\lambda_{i}(K, \bar{\Lambda}) .
\end{aligned}
$$

Remark 3.7. It is also possible to extend lower-dimensional lattices to lattices of full rank by adjoining "sufficiently large" vectors, i.e., for each $\Lambda_{i}$ of rank $n_{i}$ choose linearly independent $z_{i, n_{i}+1}, \ldots, z_{i, n} \in \Lambda \backslash \Lambda_{i}$ and consider the lattice $\bar{\Lambda}_{i}$ spanned by $\Lambda_{i}$ and $z_{i, n_{i}+1}, \ldots, z_{i, n}$. If $z_{i, j}$ are such that $\lambda_{j}\left(K, \bar{\Lambda}_{i}\right)$ is very large for $j>n_{i}$, one can apply the results from Section 3 to the collection $\bar{\Lambda}_{i}, 1 \leq i \leq s$. However, the bounds obtained in this way are in general weaker, with one exception in the case $s=1$ for the bound on $\lambda_{1}\left(K, \Lambda \backslash \Lambda_{1}\right)$. Here we get

$$
\lambda_{1}\left(K, \Lambda \backslash \Lambda_{1}\right) \leq \frac{2^{n} \operatorname{det} \Lambda}{\lambda_{1}\left(K, \Lambda_{1}\right)^{n-1} \operatorname{vol} K}+\lambda_{1}(K, \Lambda)
$$

for $\Lambda_{1} \subsetneq \Lambda$ with $\operatorname{rg} \Lambda_{1}<n$, which improves on Theorem 1.1.

\section{Acknowledgement}

We thank the anonymous referee for very helpful and valuable comments, corrections and suggestions.

\section{References}

[Ball 1991] K. Ball, “The plank problem for symmetric bodies”, Invent. Math. 104:3 (1991), 535-543. MR 92c:52003 Zbl 0702.52003

[Ball and Pajor 1990] K. Ball and A. Pajor, "Convex bodies with few faces", Proc. Amer. Math. Soc. 110:1 (1990), 225-231. MR 90m:52011 Zbl 0704.52003

[Bárány et al. 2001] I. Bárány, G. Harcos, J. Pach, and G. Tardos, "Covering lattice points by subspaces”, Period. Math. Hungar. 43:1-2 (2001), 93-103. MR 2002d:11076 Zbl 1062.11043

[Betke et al. 1993] U. Betke, M. Henk, and J. M. Wills, "Successive-minima-type inequalities", Discrete Comput. Geom. 9:2 (1993), 165-175. MR 93j:52026 Zbl 0771.52007

[Bezdek and Hausel 1994] K. Bezdek and T. Hausel, "On the number of lattice hyperplanes which are needed to cover the lattice points of a convex body”, pp. 27-31 in Intuitive geometry (Szeged, 
1991), edited by K. Böröczky and G. Fejes Tóth, Colloq. Math. Soc. János Bolyai 63, North-Holland, Amsterdam, 1994. MR 97a:52026 Zbl 0823.52006

[Bezdek and Litvak 2009] K. Bezdek and A. E. Litvak, "Covering convex bodies by cylinders and lattice points by flats", J. Geom. Anal. 19:2 (2009), 233-243. MR 2011c:52016 Zbl 1175.52008

[Blömer and Naewe 2007] J. Blömer and S. Naewe, "Sampling methods for shortest vectors, closest vectors and successive minima", pp. 65-77 in Automata, languages and programming, edited by L. Arge et al., Lecture Notes in Comput. Sci. 4596, Springer, Berlin, 2007. MR 2009k:68087 Zbl 1171.11328

[Bombieri and Vaaler 1983] E. Bombieri and J. Vaaler, "On Siegel's lemma", Invent. Math. 73:1 (1983), 11-32. MR 85g:11049a Zbl 0533.10030

[Fukshansky 2006a] L. Fukshansky, "Integral points of small height outside of a hypersurface", Monatsh. Math. 147:1 (2006), 25-41. MR 2006i:11074 Zbl 1091.11024

[Fukshansky 2006b] L. Fukshansky, "Siegel's lemma with additional conditions", J. Number Theory 120:1 (2006), 13-25. MR 2007f:11026 Zbl 1192.11018

[Gaudron 2009] É. Gaudron, "Géométrie des nombres adélique et lemmes de Siegel généralisés", Manuscripta Math. 130:2 (2009), 159-182. MR 2010i:11102 Zbl 1231.11076

[Gaudron and Rémond 2012a] É. Gaudron and G. Rémond, "Lemmes de Siegel d'évitement", Acta Arith. 154:2 (2012), 125-136. MR 2945657 Zbl 1266.11080

[Gaudron and Rémond 2012b] É. Gaudron and G. Rémond, "Polarisations et isogénies", preprint, 2012, http://math.univ-bpclermont.fr/ gaudron/art13.pdf.

[Grötschel et al. 1993] M. Grötschel, L. Lovász, and A. Schrijver, Geometric algorithms and combinatorial optimization, 2nd ed., Algorithms and Combinatorics 2, Springer, Berlin, 1993. MR 95e:90001 Zbl 0837.05001

[Gruber 2007] P. M. Gruber, Convex and discrete geometry, Grundlehren der Mathematischen Wissenschaften 336, Springer, Berlin, 2007. MR 2008f:52001 Zbl 1139.52001

[Gruber and Lekkerkerker 1987] P. M. Gruber and C. G. Lekkerkerker, Geometry of numbers, 2nd ed., North-Holland Mathematical Library 37, North-Holland, Amsterdam, 1987. MR 88j:11034 Zbl 0611.10017

[Henze 2013] M. Henze, "A Blichfeldt-type inequality for centrally symmetric convex bodies", Monatsh. Math. 170:3-4 (2013), 371-379. MR 3055793 Zbl 06176422

[Vaaler 1979] J. D. Vaaler, "A geometric inequality with applications to linear forms", Pacific J. Math. 83:2 (1979), 543-553. MR 81d:52007 Zbl 0465.52011

[Vaaler 2003] J. D. Vaaler, "The best constant in Siegel's lemma”, Monatsh. Math. 140:1 (2003), 71-89. MR 2004j:11072 Zbl 1034.11038

Received February 19, 2013. Revised May 17, 2013.

\section{MARTIN HENK}

martin.henk@ovgu.de

CARSTEN THIEL

carsten.thiel@gmail.com

FAKULTÄT FÜR MATHEMATIK

OtTO-VON-GUERICKE UNIVERSITÄT MAGDEbURG

UNIVERSITÄTSPLATZ 2

D-39106 MAGDEBURG

GERMANY 


\title{
PACIFIC JOURNAL OF MATHEMATICS
}

\author{
msp.org/pjm
}

Founded in 1951 by E. F. Beckenbach (1906-1982) and F. Wolf (1904-1989)

\section{EDITORS}

Don Blasius (Managing Editor)

Department of Mathematics

University of California

Los Angeles, CA 90095-1555

blasius@math.ucla.edu

\author{
Paul Balmer \\ Department of Mathematics \\ University of California \\ Los Angeles, CA 90095-1555 \\ balmer@math.ucla.edu \\ Robert Finn \\ Department of Mathematics \\ Stanford University \\ Stanford, CA 94305-2125 \\ finn@math.stanford.edu \\ Sorin Popa \\ Department of Mathematics \\ University of California \\ Los Angeles, CA 90095-1555 \\ popa@math.ucla.edu
}

\author{
Vyjayanthi Chari \\ Department of Mathematics \\ University of California \\ Riverside, CA 92521-0135 \\ chari@math.ucr.edu \\ Kefeng Liu \\ Department of Mathematics \\ University of California \\ Los Angeles, CA 90095-1555 \\ liu@math.ucla.edu \\ Jie Qing \\ Department of Mathematics \\ University of California \\ Santa Cruz, CA 95064 \\ qing@ cats.ucsc.edu
}

\section{PRODUCTION}

Silvio Levy, Scientific Editor, production@msp.org

\section{SUPPORTING INSTITUTIONS}

ACADEMIA SINICA, TAIPEI

CALIFORNIA INST. OF TECHNOLOGY

INST. DE MATEMÁTICA PURA E APLICADA

KEIO UNIVERSITY

MATH. SCIENCES RESEARCH INSTITUTE

NEW MEXICO STATE UNIV.

OREGON STATE UNIV.

\author{
STANFORD UNIVERSITY \\ UNIV. OF BRITISH COLUMBIA \\ UNIV. OF CALIFORNIA, BERKELEY \\ UNIV. OF CALIFORNIA, DAVIS \\ UNIV. OF CALIFORNIA, LOS ANGELES \\ UNIV. OF CALIFORNIA, RIVERSIDE \\ UNIV. OF CALIFORNIA, SAN DIEGO \\ UNIV. OF CALIF., SANTA BARBARA
}

\author{
Daryl Cooper \\ Department of Mathematics \\ University of California \\ Santa Barbara, CA 93106-3080 \\ cooper@math.ucsb.edu \\ Jiang-Hua Lu \\ Department of Mathematics \\ The University of Hong Kong \\ Pokfulam Rd., Hong Kong \\ jhlu@maths.hku.hk \\ Paul Yang \\ Department of Mathematics \\ Princeton University \\ Princeton NJ 08544-1000 \\ yang@math.princeton.edu
}

These supporting institutions contribute to the cost of publication of this Journal, but they are not owners or publishers and have no responsibility for its contents or policies.

See inside back cover or msp.org/pjm for submission instructions.

The subscription price for 2014 is US $\$ 410 /$ year for the electronic version, and \$535/year for print and electronic.

Subscriptions, requests for back issues and changes of subscribers address should be sent to Pacific Journal of Mathematics, P.O. Box 4163, Berkeley, CA 94704-0163, U.S.A. The Pacific Journal of Mathematics is indexed by Mathematical Reviews, Zentralblatt MATH, PASCAL CNRS Index, Referativnyi Zhurnal, Current Mathematical Publications and Web of Knowledge (Science Citation Index).

The Pacific Journal of Mathematics (ISSN 0030-8730) at the University of California, c/o Department of Mathematics, 798 Evans Hall \#3840, Berkeley, CA 94720-3840, is published twelve times a year. Periodical rate postage paid at Berkeley, CA 94704, and additional mailing offices. POSTMASTER: send address changes to Pacific Journal of Mathematics, P.O. Box 4163, Berkeley, CA 94704-0163.

PJM peer review and production are managed by EditFLOW ${ }^{\circledR}$ from Mathematical Sciences Publishers.

\section{PUBLISHED BY}

\section{mathematical sciences publishers \\ nonprofit scientific publishing}

http://msp.org/

(C) 2014 Mathematical Sciences Publishers 


\section{PACIFIC JOURNAL OF MATHEMATICS}

Volume $269 \quad$ No. $2 \quad$ June 2014

Totaro's question for simply connected groups of low rank

257

JODI BLACK and RAMAN PARIMALA

Uniform hyperbolicity of the curve graphs

269

BRIAN H. BOWDITCH

Constant Gaussian curvature surfaces in the 3-sphere via loop groups

281

DAVID BRANDER, JUN-ICHI INOGUCHI and SHIMPEI KOBAYASHI

On embeddings into compactly generated groups

305

PIERRE-EMmanuel CAPRACE and Yves CORNULIER

Variational representations for $N$-cyclically monotone vector fields

ALFRED GALICHON and NASSIF GHOUSSOUB

Restricted successive minima

341

MaRTIN HenK and CARSTEN THIEL

Radial solutions of non-Archimedean pseudodifferential equations

355

ANATOLY N. KochubeI

A Jantzen sum formula for restricted Verma modules over affine Kac-Moody

algebras at the critical level

JOHANNES KÜBEL

Notes on the extension of the mean curvature flow

YAN LENG, ENTAO ZHAO and HaORAN ZHAO

Hypersurfaces with prescribed angle function

Henrique F. DE Lima, ERAldo A. Lima JR. and Ulisses L. Parente

Existence of nonparametric solutions for a capillary problem in warped products

JoRGE H. LiRA and GABRIELA A. WANDERLEY

A counterexample to the simple loop conjecture for $\operatorname{PSL}(2, \mathbb{R})$

425

KATHRYN MANN

Twisted Alexander polynomials of 2-bridge knots for parabolic representations

TAKAYUKI MORIFUJI and ANH T. TRAN

Schwarzian differential equations associated to Shimura curves of genus zero

FANG-TING TU

Polynomial invariants of Weyl groups for Kac-Moody groups 